\title{
Workshop der European Association of Dacryology (EAD) (Präs.: Van Bjisterveld) und ÖOG, Salzburg 2003
}

Zum ersten Mal fand im Rahmen der Jahrestagung der Österreichischen Gesellschaft für Ophthalmologie in Salzburg ein gemeinsames Mini-Symposium zum Trockenen Auge statt. Mit Grußbotschaften des Sekretärs Prof. Dr. Van Setten (Stockholm, Karolinska-Institut) und vom Vorstand der ÖOG wurde die halbtägige Sitzung eröffnet. Vertreter der österreichischen und deutschen Forschungsgruppen rund um das Thema ,Trockenes Auge“ behandelten sowohl Ätiologie, Diagnostik, als auch therapeutische Richtlinien. Kurze Zusammenfassungen werden anschließend wiedergegeben.
Darüber hinaus hat Dr. Markovic (Wien) seine Arbeit über die Anamnese beim Trockenen Auge als Originalarbeit zur Verfügung gestellt. Mit einem Überblick zur Therapie darf ich die Sammlung abschließen.

Nicht zuletzt möchte ich mich bei allen bedanken, die zum Gelingen der Veranstaltung beigetragen haben: den Sponsoren Novartis und Croma, der Österreichischen Gesellschaft für Ophthalmologie, den Mitarbeitern der Ambulanz für Trockene Augen, insbesonders Frau Dr. Funk, sowie den Salzburger Organisatoren!

Dr. Johannes Nepp

\section{Neue Aspekte zur Pathophysiologie des Trockenen Auges}

\section{T. Kaercher}

Augenklinik des Städtischen Klinikums Ludwigshafen

An der Augenoberfläche finden sich Proteine und Peptide, welche die Existenz eines unspezifischen Abwehrsystems wahrscheinlich machen. Dieses Abwehrsystem verhindert das Auftreten von Benetzungsstörungen und Entzündungen. Die Substanzklassen der Lipocaline, Defensine und Collectine scheinen die wesentlichen Bestandteile dieses Abwehrsystems zu sein.

Die Lipocaline machen etwa 20\% der Gesamtproteine der Träne aus. Im Gegensatz zu dem häufig genannten Lactoferrin und Lysozym sind die Lipocaline negativ geladen. Ihr Molekulargewicht liegt bei 17.000 . Sie weisen eine hohe Sequenzhomologie von $25-30 \%$ auf. Interessant ist, dass eine Identität zwischen den Tränendrüsen-Lipocalinen und den Lipocalinen der Von-Ebnerschen Speicheldrüsen nachgewiesen werden konnte. Während die Aufgabe der Lipocaline in den Speicheldrüsen im Binden und anschließenden Abspülen von Fremdpartikeln besteht, ist deren Funktion an der Augenoberfläche noch unklar. Ihre wesentliche Funktion scheint die Bindung kleiner Moleküle zu sein, eventuell ist der RetinolTransport lipocalinabhängig. Auch wird eine Modulation der Immunantwort durch Lipocaline für möglich gehalten; damit wird ihnen eine epithelprotektive Funktion zugeschrieben.
Die Defensine sind eine Peptidklasse, die sich in der Tränendrüse und im Tränenfilm nachweisen lässt. Defensine bestehen aus 29-35 Aminosäuren, sie finden sich in einer $\alpha$ und $\beta$-Form. Defensine haben Anteil am Spannung-Aufbau an Plasma-Membranen. Sie sind ebenso beteiligt an der zellulären Immunantwort, möglicherweise haben sie auch Einfluss auf die Epithelzellmitose. Es besteht die Aussicht, dass rekombinante Defensine an der Augenoberfläche therapeutisch eingesetzt werden können.

Collectine sind eine Klasse von 4 Surfactant-Proteinen, die bislang nur in der Lunge und in der Leber bekannt waren. Kürzlich konnte ein Collectin auch in der Tränendrüse der Maus nachgewiesen werden. Die Funktion der Collectine A und $\mathrm{D}$ besteht in der Bindung von Bakterien. Die Collectine $B$ und $C$ sind hingegen für Spreitz- und Transportvorgänge verantwortlich.

Durch das Zusammenspiel der 3 genannten Substanzgruppen ist es möglich, an der Augenoberfläche Fremdpartikel und Bakterien zu binden, abzutransportieren und damit eine Immunreaktion zu verhindern. Sollte sich dieser Mechanismus als unzureichend erweisen, so kommt es $\mathrm{zu}$ einer 2. Stufe der Abwehrreaktion und zum Auftreten von Be- 